to at least three or more stressful early life events. Alterations of this nature may consequently predispose such individuals to emotional and cognitive dysfunctions. These findings may have implications for understanding the pathways to psychiatric disorders of cognition and mood, and may provide some guide to the tailoring of treatment according to the patient's genetic/endophenotypic profile.

$10-04$

\section{Identifying markers of negative mood: the gender-specific influence of COMT and MAO-A polymorphisms on emotion processing}

\section{SA Kuan', JM Gatt', C Dobson-Stone ${ }^{2}$, RH Paul ${ }^{3}$, PR Schofield ${ }^{2}$, E Gordon ${ }^{4}$, LM Williams ${ }^{1}$}

'The Brain Dynamics Centre, Westmead Millennium Institute, Westmead Hospital and Western Clinical School, University of Sydney, Australia; ${ }^{2}$ Prince of Wales Medical Research and Garvan Institutes, Australia; ${ }^{3}$ Brown Medical School, Rhode Island, USA; and ${ }^{4}$ The Brain Resource International Database

(The Brain Resource Company), Australia

Background: Our integrative neuroscience model of emotion processing proposes that the effects of genetic polymorphisms on emotional function and risk for disorders of negative affect may vary with gender. The COMT Met allele has been related to anxiety traits (in women), while MAO-A genotypes have been linked to anxiety and phobic disorders (in women) and increased aggression (in men). Using a facial emotion perception task, we examined the role of neuroimaging endophenotypes in the association between COMT, MAO-A and negative mood, and the moderating effects of gender.

Methods: About 273 healthy subjects from the Brain Resource International Database provided data from cheek swabs (for genotyping). We assessed mood and temperament (using DASS and NEO), and emotionrelated brain function (using event-related potential recording).

Results: COMT heterozygotes (V/M) were associated with higher neuroticism, and reduced and delayed neural responses to emotion in women. By contrast, while the MAO-A genotype showed no direct effects on negative mood, the high-activity alleles were associated with faster and greater responses to emotion in men.

Conclusions: The gender-related dissociation in the impact of COMT and MAO-A on emotion processing and negative mood suggests that these variants contribute to the differential expression of mood disorders in men and women. Integrative genotype-endophenotype makers may offer promise as a tool to aid in early identification of vulnerability to mood disorder and the selection of optimal treatments.

$10-05$

\section{Genotypes and neural binding in negative affect: the contribution of genetic polymorphisms to $40 \mathrm{~Hz}$ gamma phase synchrony \\ JM Gatt' ${ }^{1}$, S Kuan'1, C Dobson-Stone ${ }^{2}$, RH Paul ${ }^{3}$, PR Schofield ${ }^{2}$, E Gordon ${ }^{4}$, LM Williams ${ }^{1}$}

\footnotetext{
'The Brain Dynamics Centre, Westmead Millennium Institute, Westmead Hospita and Western Clinical School, University of Sydney, Australia; 2 Prince of Wales Medical Research and Garvan Institutes, Australia; ${ }^{3}$ Brown Medical School, Rhode Island, USA; and ${ }^{4}$ The Brain Resource International Database (The Brain Resource Company), Australia
}

Objective: Binding of diverse neural activity is essential for complex cognitive and emotional functions. There is increasing evidence for the contribution of genetic polymorphisms to these functions, but their role in neural binding is unknown. We explored differences in $40 \mathrm{~Hz}$ gamma synchrony (an index of high-frequency binding) according to COMT Val108/158Met, BDNF Val66Met, MAOA and 5HTT-LPR genotypes, and their combined role in negative mood.

Methods: About 155 healthy subjects from the Brain Resource International Database provided cheek swabs (for genotyping) and were assessed for level of depressed mood and anxiety and early life stress. Gamma phase synchrony was extracted from EEG recordings during perception of facial emotion stimuli, pertinent to eliciting biases in negative affect states.

Results: Reduced synchrony to emotional expressions was related to higher depression, and enhanced synchrony to higher anxiety, suggesting distinct biases in binding with these aspects of mood. Consistent with this pattern, the 5HTT-LPR SS allele was linked to reduced frontal and parieto-occipital synchrony to fear with higher stress. The COMT Met allele was linked to similarly reduced frontotemporal synchrony to fear and happiness. By contrast, the BDNF Met allele was related to enhanced synchrony to both fear and happiness with higher stress, suggesting heightened sensitivity to emotion. Synchrony was also enhanced, right parietally and frontotemporally, for the MAOA low-activity allele, particularly later in the time course.

Conclusion: Polymorphisms that influence brain function may have distinct effects on neural binding associated with processing salient signals of emotion, 
and may contribute to behavioural states of depressed mood and anxiety.

\section{'Integrative Neuroscience' and Psychiatry: Identifying Cognitive, Affective and Brainwave Markers of Psychiatric Disorder}

\section{Williams}

The Brain Dynamics Centre, Westmead Millennium Institute, Westmead Hospital and Discipline of Psychological Medicine, University of Sydney, Australia

\section{Overview}

Multidisciplinary efforts have begun to encourage a freer exchange of information - resulting in a more 'integrative neuroscience' across disciplines and theoretical models in psychiatry. This symposium outlines the potential insights into major psychiatric disorders from the first entirely standardized and centralized database, which brings together cognitive, affective, brain function and genetic measures. It contains 5000 healthy subjects $(6-100$ years) and growing psychiatric groups. With these multimodal and standardized data sets, we have identified objective markers that distinguish each disorder and that predict real-life functional outcomes. In this symposium, we outline markers for first-episode schizophrenia, attention deficit hyperactivity disorder, depression and Alzheimer's dementia.

$11-01$

\section{Identifying cognitive, affective and neural synchrony markers which predict real-world functional outcome in first-episode schizophrenia: an integrative neuroscience approach}

\section{Williams' ${ }^{1}$, J Whitford', BJ Liddell ${ }^{1,2}$, D Alexander ${ }^{1,2}$, G Flynn ${ }^{1,3}$, W Wong ${ }^{1,3}$, P Das ${ }^{1,4}$, AWF Harris', E Gordon ${ }^{1,2}$}

'The Brain Dynamics Centre, Westmead Millennium Institute, Westmead Hospital and Western Clinical School, University of Sydney, Australia; ${ }^{2}$ Brain Resource International Database, Brain Resource Company; ${ }^{3}$ Early Psychosis Intervention Program, Liverpool Hospital; and ${ }^{4}$ Neuroscience Institute of Schizophrenia and Allied Disorders (NISAD), New South Wales, Australia

Background: In addition to psychotic symptoms, first-episode schizophrenia (FES) is characterized by profound difficulties in cognition and social and emotional functions.

We examined whether cognitive markers predict realworld functional outcome in FES and whether this prediction is enhanced by social-cognitive markers. We also examined the relationship between these markers and lack of neural synchrony in FES, in the context of our integrative neuroscience model of impaired neural coordination of salient and task-relevant information in this condition.

Method: We tested patients with FES (within 3 months of service contact) using the standardized Brain Resource International Database cognitive battery and tests of social and emotional function (including DASS, NEO-FFI). Neural synchrony was extracted from EEG recorded to corresponding tasks. Assessments of symptoms and functional outcome included PANSS, SOFAS, and World Health Organisation Quality of Life Scale.

Results: General cognitive markers predicted negative symptom severity, with the greatest contribution from poor verbal function, then visuospatial executive functions. Poorer executive function also predicted poorer social and occupational outcome and quality of life. This predictive relationship was significantly improved by the addition of the social-cognitive marker, excessive negativity bias. These combined markers were also related to an excess of high-frequency neural synchrony in EEG recordings.

Conclusion: These findings show that 1) markers of general cognitive dysfunction predict real-world functional outcome in FES, 2) predictive power is enhanced by taking social and emotional aspects of cognition into account and 3) impairments in neural binding and coordination of salient stimuli may underlie these functional difficulties.

\section{$11-02$}

\section{Identifying affective markers within an integrative neuroscience model of depression}

\author{
D Mathersul', A Kemp ${ }^{1,2}$, P Hopkinson ${ }^{1,3}$, E Gordon ${ }^{1,3}$ \\ 'The Brain Dynamics Centre, Westmead Millennium Institute, Westmead Hospital \\ and Western Clinical School, University of Sydney, Australia; ${ }^{2}$ Psychological \\ Medicine, University of Sydney; and ${ }^{3} B r a i n$ Resource International Database, \\ Brain Resource Company, Sydney, Australia
}

Background: Our integrative model of depression focuses on disturbances in affective and cognitive function, which contribute to clinical depression. Endophenotypes for depression include disturbances 